\title{
Cornual Rupture of Uterus
}

\author{
Lt Col Y Singh", Col VRR Chari (Retd) ${ }^{+}$, Col SK Basu (Retd)
}

MJAFI 2008; $64: 81$

Key Words: Cornual rupture; Post caesarean

\section{Introduction}

U terine rupture during the course of labour is a well documented complication and the majority of cases occur in women with scarred uteri. However, a rupture that occurs in areas of presumably normal tissue instead of scar tissue is extremely rare. We present a case of cornual rupture in a post-caesarean pregnancy.

\section{Case Report}

A 28 year old patient $\left(\mathrm{G}_{2} \mathrm{P}_{1}\right)$, with a previous lower segment caesarean section (LSCS) done for foetal distress, was admitted at 38 weeks of gestation for trial of vaginal delivery. There was no history of dilatation \& curettage (D\&C), medical termination of pregnancy (MTP), hysteroscopy, myomectomy or intra uterine contraceptive device (IUCD) insertion. Her routine investigations were within normal limits and an ultrasound at the 37th week of gestation showed normal foetal growth with a fundal presentation. After hospitalisation, Bishop scores showed favourable conditions for vaginal delivery. Two days after admission, the patient went into spontaneous labour. Labour progressed smoothly. During the process, neither oxytocin nor prostaglandin augmentation were administered. The uterine contractions were neither overtly strong, nor abnormally frequent. In view of her previous caesarean and acute foetal distress, the second stage was cut down by applying a vacuum. She delivered a still-born female weighing $2.9 \mathrm{~kg}$, and there was no postpartum haemorrhage. Immediately after delivery, the patient had acute abdominal pain, accompanied by a sudden drop in blood pressure. The abdomen was diffusely tender, with significant rebound tenderness and muscle guarding. A diagnosis of internal bleeding associated with hemorrhagic shock was suspected.

The patient underwent emergency laparotomy, which revealed a large haemoperitoneum ( $4000 \mathrm{ml}$ of blood) and a three centimeter long rupture in the cornual region of the uterine wall. The previous lower segment scar was healthy and no other uterine deformity was noted. A primary repair of the uterus in two layers was performed. After appropriate replacement of intra-operative and post-operative blood loss, the patient made an uneventful recovery. Her preoperative haemoglobin was $11.8 \mathrm{gm} \%$ and the postoperative value after giving six units of blood was $7 \mathrm{gm} \%$.

\section{Discussion}

Vaginal birth after caesarean section (VBAC) is now a well accepted procedure in pregnant women who do not have any contraindications. Rupture occurring in presumably normal tissue, as opposed to scarred areas, is extremely rare [1]. There are few case reports of spontaneous rupture without significant risk factors $[2,3]$. Most reports demonstrated that underlying pathological changes could be anticipated in the presence of certain conditions;multiparity, uterine anomalies like uterine diverticulae and bicornuate uteri, placenta percreta, arteriovenous malformation, endometriosis, precipitous labour and obstructed labour. However our patient was free from any of the above risk factors which might have contributed to spontaneous uterine rupture.

We could not find a reasonable explanation as to why the uterus ruptured in that area. The possibility of any underlying pathological changes could not be ruled out since no biopsy was taken during emergency laparotomy.

\section{Conflicts of Interest}

None identified

\section{References}

1. Hawe JA, Olah KS. Posterior uterine rupture in a patient with a lower segment cesarean scar complicating prostaglandin induction of labour. British Journal Obstet Gynecol 1997; 104: 857-8.

2. Langton J F, Kumar K. Spontaneous rupture of an unscarred gravid uterus of 32 weeks gestation. Hum Reprod 1997; 12: 2066-7.

3. Wang PH, Chao HT. Primary repair after cornual rupture occurred in a 21 weeks gestation pregnant woman. Human Reprod 1999; 14: 1894-5.

${ }^{*}$ Reader ( Department of Obstetrics and Gynaecology), AFMC, Pune. ${ }^{+}$Ex-Senior Advisor (Anaesthesiology), 158 Base Hospital C/o 99APO. "Ex-Senior Advisor (Obstetrics and Gynaecology), Command Hospital (EC), Kolkata. ${ }^{* *}$ Medical Officer, 14 BRTF, C/o 99 APO. 